\title{
IRRATIONAL POWER SERIES ${ }^{1}$
}

\section{MORRIS NEWMAN}

In this note the following theorem will be proved:

Theorem 1. Let $\alpha$ be a real number. Let $g$ be a polynomial of degree $p \geqq 1$. Define

$$
G=\sum_{n=0}^{\infty} g([n \alpha]) x^{n}
$$

Then $G$ is a rational function of $x$ if and only if $\alpha$ is a rational number.

As usual, $[x]$ denotes the integral part of $x$ and $\{x\}$ the fractional part of $x$, so that $x=[x]+\{x\}$. We first prove

LEMMA 1. Let $\alpha$ be a real irrational number, and let $R$ be a finite set of non-integral real numbers. Then there are infinitely many positive integers $m$ such that

$$
[\{m \alpha\}+r]=[r], \quad r \in R,
$$

and infinitely many positive integers $n$ such that

$$
[\{n \alpha\}+r]=1+[r], \quad r \in R .
$$

Proof. We have that (1) is completely equivalent to

$$
0 \leqq\{m \alpha\}+\{r\}<1, \quad r \in R
$$

and that (2) is completely equivalent to

$$
0 \leqq\{n \alpha\}+\{r\}-1<1, \quad r \in R .
$$

Since $R$ consists of a finite number of nonintegral reals, there are numbers $a$ and $b$ such that

$$
0<a \leqq\{r\} \leqq b<1, \quad r \in R .
$$

Therefore we need only choose $m$ and $n$ so that

$$
\begin{aligned}
& \{m \alpha\}<1-b, \\
& \{n \alpha\} \geqq 1-a .
\end{aligned}
$$

Since $\alpha$ is irrational the numbers $\{\alpha\},\{2 \alpha\}, \cdots$ are uniformly

Received by the editors October 13, 1959.

1 The preparation of this paper was supported (in part) by the Office of Naval Research. 
distributed in the unit interval, and so (3) and (4) each have infinitely many positive integral solutions. This completes the proof of the lemma.

We go on now to the proof of Theorem 1. Assume first that $\alpha$ is irrational. If $G$ is rational, then polynomials $A$ and $B$ of degrees $a$ and $b$ respectively exist such that $G=B / A$; and there is no loss of generality in assuming that

$$
A=1-c_{1} x-c_{2} x^{2}-\cdots-c_{a} x^{a} .
$$

Then $A G=B$, and comparing coefficients we find that

$$
g([n \alpha])=\sum_{r=1}^{a} g([n \alpha-r \alpha]) c_{r}, \quad n \geqq a, n>b .
$$

Now for $1 \leqq r \leqq a$,

$$
\lim _{n \rightarrow \infty} \frac{g([n \alpha-r \alpha])}{g([n \alpha])}=\lim _{n \rightarrow \infty} \frac{[n \alpha-r \alpha]^{p}}{[n \alpha]^{p}}=1 .
$$

This implies that

$$
\sum_{r=1}^{a} c_{r}=1
$$

Hence

$$
\sum_{r=1}^{a}(g([n \alpha-r \alpha])-g([n \alpha])) c_{r}=0 .
$$

We have $[n \alpha-r \alpha]=[\{n \alpha\}-r \alpha]+[n \alpha]$ and so

$$
g([n \alpha-r \alpha])-g([n \alpha])=\sum_{k=1}^{p} \frac{g^{(k)}([n \alpha])}{k !}[\{n \alpha\}-r \alpha]^{k} .
$$

Thus

$$
\sum_{r=1}^{a}[\{n \alpha\}-r \alpha] c_{r}+\sum_{r=1}^{a} \sum_{k=2}^{p} \frac{g^{(k)}([n \alpha])}{k ! g^{\prime}([n \alpha])}[\{n \alpha\}-r \alpha]^{k} c_{r}=0 .
$$

If $p=1$ the last sum is vacuous, and if $p \geqq 2$ we have

$$
\lim _{n \rightarrow \infty} \frac{g^{(k)}([n \alpha])}{g^{\prime}([n \alpha])}[\{n \alpha\}-r \alpha]^{k}=0, \quad 2 \leqq k \leqq p,
$$

since the numbers $[\{n \alpha\}-r \alpha]^{k}$ are uniformly bounded for all $n$. In either case, we deduce that 


$$
\lim _{n \rightarrow \infty} \sum_{r=1}^{a}[\{n \alpha\}-r \alpha] c_{r}=0 .
$$

Now the numbers $-r \alpha$ are nonintegral for $1 \leqq r \leqq a$. Thus Lemma 1 applies and choosing sequences for which (1) and (2) hold, we obtain

$$
\begin{aligned}
\sum_{r=1}^{a}[-r \alpha] c_{r} & =0, \\
\sum_{r=1}^{a}(1+[-r \alpha]) c_{r} & =0 .
\end{aligned}
$$

But (8) and (9) together imply that

$$
\sum_{r=1}^{a} c_{r}=0
$$

which contradicts (6). Thus we have proved that if $\alpha$ is irrational then $G$ is not a rational function.

Suppose now that $\alpha$ is rational, and write $\alpha=c / d$ where $d>0$, $(c, d)=1$. Then

$$
\begin{aligned}
G & =\sum_{n=0}^{\infty} g([n \alpha]) x^{n}=\sum_{r=0}^{d-1} \sum_{m=0}^{\infty} g\left(m c+\left[r \frac{c}{d}\right]\right) x^{m d+r} \\
& =\sum_{r=0}^{d-1} \sum_{m=0}^{\infty} \sum_{k=0}^{p} \frac{g^{(k)}\left(\left[\frac{r c}{d}\right]\right)}{k !}(m c)^{k} x^{m d+r} \\
& =\sum_{r=0}^{d-1} \sum_{k=0}^{p} \frac{g^{(k)}\left(\left[\frac{r c}{d}\right]\right)}{k !} c^{k} x^{r} \sum_{m=0}^{\infty} m^{k} x^{m d} .
\end{aligned}
$$

But it is well-known that

$$
\sum_{m=0}^{\infty} m^{k} x^{m}
$$

is rational: Indeed,

$$
\sum_{m=0}^{\infty} m^{k} x^{m}=\left(x \frac{d}{d x}\right)^{k}(1-x)^{-1} .
$$

Hence $G$ is also rational, and the proof of Theorem 1 is complete.

An interesting result that Theorem 1 implies is 
Theorem 2. Suppose that $\alpha>0$. Define

$$
F=\sum_{n=0}^{\infty} x^{[n \alpha]} .
$$

Then $F$ is a rational function if and only if $\alpha$ is rational.

Proof. Suppose that $\alpha$ is irrational. Let $\chi(n)$ be the number of solutions of $n=[t \alpha]$ in non-negative integers $t$. Then obviously,

$$
F=\sum_{n=0}^{\infty} \chi(n) x^{n} .
$$

But $\chi(n)$ is just the number of integers $t$ satisfying $n \leqq t \alpha<n+1$; and since $\alpha$ is irrational, this implies that

$$
\chi(n)=\left[\frac{n+1}{\alpha}\right]-\left[\frac{n}{\alpha}\right] .
$$

Thus

$$
F=(1-x) \sum_{n=1}^{\infty}\left[\frac{n}{\alpha}\right] x^{n-1}
$$

and the conclusion (for $\alpha$ irrational) follows from Theorem 1 .

Suppose now that $\alpha$ is rational, and write (as before) $\alpha=c / d$ where $d>0,(c, d)=1$. Then

$$
\begin{aligned}
F & =\sum_{n=0}^{\infty} x^{[n \alpha]}=\sum_{r=0}^{d-1} \sum_{m=0}^{\infty} x^{m c+[r(c / d)]} \\
& =\left(1-x^{c}\right)^{-1} \sum_{r=0}^{d-1} x^{[r(c / d)]} .
\end{aligned}
$$

Hence $F$ is rational, and the proof of Theorem 2 is complete.

National Bureau of Standards 